\title{
ON STATES IN MV-ALGEBRAS AND THEIR APPLICATIONS
}

\author{
ANATOLIJ DVUREČENSKIJ ${ }^{1}$
}

${ }^{1}$ Mathematical Institute, Slovak Academy of Sciences, Štefánikova 49, SK-814 73 Bratislava, Slovakia

E-mail: dvurecen@mat.savba.sk

In the frames of quantum structures, states are a very important notion that model probability on an algebraic structure. Already G. Boole observed that to calculate probability of event of the structure $M$, it is important to know only which two events $A$ and $B$ we can add such that if $C=A+B$, and $P$ is a probability, then $P(A+B)=P(A)+P(B)$; and the operation + is a partial one on $M$. If $M$ is a Boolean algebra, then $A+B:=A \cup B$ whenever $A \cap B=\emptyset$ or equivalently, $A \leq B^{\prime}$. Such two events $A$ and $B$ are said to be mutually excluding or summable, $[\mathrm{DvPu}]$.

Therefore, the state or finitely additive state on an algebraic structure $\left(M ;+,,^{\prime}, 0,1\right)$ is any mapping $s: M \rightarrow[0,1]$ such that (i) $s(1)=1$, and (ii) $s(a+b)=s(a)+s(b)$ whenever $a+b$ is defined in $M$. The unary operation ' is an orthogonal complement or a negation.

The basic task is how to define a state on an algebraic structure like $\left(M ; \oplus, \odot,^{*}, 0,1\right)$ or $\left(M ; \oplus, \odot,{ }^{-}, \sim, 0,1\right)$, that is, how to derive a partial operation + from the $\oplus, \odot$ ?

In more complicated structures, like orthomodular lattices and effect algebras, the most important example is the system $\mathcal{L}(H)$ of all closed subspaces of a Hilbert space $H$ or the system of all Hermitian operators $\mathcal{E}(H)$ that are between the zero operator and the identity operator. Here the $\sigma$-additive states are of the form

$$
\begin{gathered}
s_{\phi}(M)=\left(P_{M} \phi, \phi\right), M \in \mathcal{L}(H), \phi \in H, \\
s(M)=\sum_{i} \lambda_{i} s_{\phi}(M)=\operatorname{tr}\left(T P_{M}\right), M \in \mathcal{L}(H) .
\end{gathered}
$$

Gleason theorem, 1957, $3 \leq \operatorname{dim} H \leq \aleph_{0}$, [Dvu].

If $s$ is a finitely additive on $L(H)$, Aarnes [Dvu],

$$
s=\lambda s_{1}+(1-\lambda) s_{2}
$$

where $s_{1}$ is a $\sigma$-additive state on $\mathcal{L}(H)$ and $s_{2}$ a finitely additive state vanishing on each finite-dimensional subspace of $H$.

\footnotetext{
${ }^{1}$ The paper has been supported by the Center of Excellence SAS - Physics of Information - I/2/2005, the grant VEGA No. 2/6088/26 SAV, by Science and Technology Assistance Agency under the contract No. APVV-0071-06.
} 
On the other hand, each Boolean algebra has plenty finitely additive states, but there is an example of Boolean $\sigma$-algebra that has no $\sigma$ additive states. There are plenty of OML's or OMP's that are stateless.

An effect algebra, Foulis and Bennett (D-poset Kôpka and Chovanec) $[\mathrm{DvPu}]$, is a partial algebra $(M ; \oplus, 0,1)$ such that $(\mathrm{i})+$ is associative and commutative, (ii) for each $a \in M$ there is a unique $a^{\prime} \in M$ such that $a+a^{\prime}=1$, and (iii) if $a+1$ is defined in $M$ then $a=0$.

Example: Let $(G ;+, \leq)$ be a po-group, and $u>0$. Set $\Gamma(G, u)=$ $[0, u]$, and let + be the restriction of the group addition to $\Gamma(G, u)$. Then $\Gamma(G, u)$ is an effect algebra (called an interval effect algebra). Each such an effect algebra admits at least one state. For example, if $\mathcal{B}(H)$ is the system of all Hermitian operators on $H$, then $\mathcal{E}(H)=\Gamma(\mathcal{B}(H), I)$.

The Riesz decomposition property: If $c \leq a+b$ then there are $a_{1}, b_{1} \in M$ such that $a_{1} \leq a, b_{1} \leq b$ and $c=a_{1}+b_{1}$. This is equivalent if $a_{1}+a_{2}=b_{1}+b_{2}$, there are four elements $c_{11}, c_{12}, c_{21}, c_{22} \in M$ such that $a_{1}=c_{11}+c_{12}, a_{2}=c_{21}+c_{22}, b_{1}=c_{11}+c_{21}$, and $b_{2}=c_{21}+c_{22}$.

Ravindran showed that if an effect algebra $M$ satisfies RDP, then there is a unique unital interpolation po-group $(G, u)$ such that $M=$ $\Gamma(G, u),[\mathrm{DvPu}]$.

If $\left(M ; \oplus, \odot,{ }^{*}, 0,1\right)$ is an MV-algebra, then defining a partial operation + on $M$ via $a+b$ is defined iff $a \odot b=0$ (equivalently, $a \leq b^{*}$ ), and then we set $a+b=a \oplus b$. Then $(M ;+, 0,1)$ is an effect algebra with RDP.

A state $s$ is said to be extremal if from $s=\lambda s_{1}+(1-\lambda) s_{2}, 0<\lambda<1$, we have $s=s_{1}=s_{2}$. Let $\mathcal{S}(M)$ and $\mathcal{S}_{\partial}(M)$ be the system of all states, respectively extremal states, on $M$. If $\mathcal{S}(M) \neq \emptyset$, then, due to the Krein-Milman theorem

$$
\mathcal{S}(M)=\left(\operatorname{ConHull}\left(\mathcal{S}_{\partial}(M)\right)\right)^{-w} .
$$

If $s$ is state on EA $M$, then

$$
\operatorname{Ker}(s)=\{a \in M: s(a)=0\}
$$

is an ideal of $M$.

If $M$ is an MV-algebra, then a state $s$ is extremal iff $\operatorname{Ker}(s)$ is a maximal ideal on $M$. There is a one-to-one correspondence between extremal states and maximal ideals on $M$ via $s \leftrightarrow \operatorname{Ker}(s), s$ is extremal iff $s(a \oplus b)=\min (s(a)+s(b), 1) . \mathcal{S}_{\partial}(M)$ is a compact Hausdorff topological space. If $M$ is only an EA, this is not true, in general. The situation with GMV-algebras or pseudo effect algebras is similar but more complicated [Dvu3].

A tribe is a system $\mathcal{T}$ of fuzzy sets on $\Omega$ such that (i) $1_{\Omega} \in \mathcal{T}$, (ii) if $f \in \mathcal{T}$, then $1-f \in \mathcal{T}$, and (iii) if a sequence $\left\{f_{n}\right\}$ is taken from $\mathcal{T}$, then $\bigoplus_{n} f_{n}:=\min \left(\sum_{n} f_{n}, 1\right) \in \mathcal{T}$. For any family $\mathcal{F}$ of fuzzy sets on $\Omega$, there is a minimal tribe generated by $\mathcal{F}$.

Mundici [Mun], Dvurečenskij [Dvu1], Barbieri and Barbieri [BaWe]: 
Theorem 1 (Loomis-Sikorski). Every $\sigma$-complete $M V$-algebra $M$ is a $\sigma$-epimorphic image of a tribe. This tribe can be choose to be generated by extremal states on $M$.

Buhagiar, Chetcuti, Dvurečenskij, [BCD]:

Theorem 2. Every $\sigma$-complete EA $M$ with $R D P$ is a $\sigma$-epimorphic image of a $\sigma$-complete $E A$ of fuzzy sets with pointwise defined operation satisfying $R D P$.

Another approach is via state MV-algebras, Flaminio and Montagna, [FlMo]. We define a more narrow notion: an extremal state MValgebra is a system $\left(M ; \oplus, \odot,{ }^{*}, \sigma, 0,1\right)$ where $\sigma$ is a homomorphism of the MV-algebra $M$ into $M$ such that $\sigma^{2}=\sigma$. We describe all subdirectly irreducible elements of the variety of extremal state MV-algebras (Di Nola and Dvurečenskij, [DiDv]):

Theorem 3. $(M, \sigma)$ is subdirectly irreducible iff either $M$ is a subdirectly irreducible $M V$-algebra or $M=A \times B$, where $A$ is a chain $M V$ algebra, $B$ a subdirectly irreducible $M V$-algebra, and $\sigma(a, b)=(a, h(a))$, $(a, b) \in A \times B$, where $h: A \rightarrow B$ is an MV-homomorphism.

\section{REFERENCES}

[BaWe] G. Barbieri, H. Weber, Measures on clans and on MV-algebras, In: Handbook of Measure Theory, E. Pap (Editor), Elsevier Science, Amsterdam, 2002, Vol. II, pp. 911-945.

[BCD] D. Buhagiar, E. Chetcuti, A. Dvurečenskij, Loomis-Sikorski representation of monotone $\sigma$-complete effect algebras, Fuzzy Sets and Systems 157 (2006), 683-690.

[DiDv] A. Di Nola, A. Dvurečenskij, Extremal state $M V$-algebras, in prepartion.

[Dvu] A. Dvurečenskij, "Gleason's Theorem and Its Applications", Kluwer Academic Publisher, Dordrecht/Boston/London, 1993, 325+xv pp.

[DvPu] A. Dvurečenskij, S. Pulmannová, "New Trends in Quantum Structures", Kluwer Acad. Publ., Dordrecht, Ister Science, Bratislava, 2000.

[Dvu1] A. Dvurečenskij, Loomis-Sikorski theorem for $\sigma$-complete $M V$-algebras and $\ell$-groups, J. Austral. Math. Soc. Ser. A 68 (2000), 261-277.

[Dvu2] A. Dvurečenskij, Pseudo MV-algebras are intervals in $\ell$-groups, J. Austral. Math. Soc. 72 (2002), 427-445.

[Dvu3] A. Dvurečenskij, States on pseudo MV-algebras, Studia Logica 68 (2001), 301-327.

[DvVe1] A. Dvurečenskij, T. Vetterlein, Pseudoeffect algebras. I. Basic properties, Inter. J. Theor. Phys. 40 (2001), 685-701.

[FlMo] T. Flaminio, F. Montagna, An algebraic approach to states on $M V$ algebras, In: V. Novák (ed.), Fuzzy Logic 2, Proc. of the 5th EUSFLAT Conf., Sept. 11-14, 2007, Ostrava, vol. II, pp. 201-206.

[Mun] D. Mundici, Tensor products and the Loomis-Sikorski theorem for MValgebras, Advan. Appl. Math. 22 (1999), 227-248. 